\title{
Investment Portfolio Establishment Based on Index Model Linked with Different Constraints
}

\author{
Shaoyu Ding ${ }^{1, *, a, \uparrow}$, Heming Ma ${ }^{2, *},{ }^{*}, \dagger$, Shiyue Zhang ${ }^{3, *, c, \dagger}$ \\ ${ }^{1}$ Art and Science, Emory University, 30322, Atlanta, United States \\ ${ }^{2}$ Internation Business School, Jinan University, 300280, Zhuhai, China \\ ${ }^{3}$ D'Amore-McKim School of Business, Northeastern University, 02115, Boston, United States \\ *Corresponding author. Email: ${ }^{a}$ shaoyu.ding@emory.edu \\ ${ }^{\dagger}$ These authors contributed equally.
}

\begin{abstract}
After Markowitz Model was invented, people continue to find series of problems about implementing the model to real world and Index model was introduced to solve these problems. In this paper, we use index model to form minimum variance portfolios and maximum Sharpe portfolios for series of stocks and S\&P 500 index. To see how model performs and provide advice to investors under real-world regulations, we implement index model under 5 different constraints and plot minimum variance frontiers, minimum return frontiers and efficient frontiers. The paper compares results for index model under different constraints, and further analysis suggests that regulations aiming at preventing investors from having extreme position performs better than those banning shorting.
\end{abstract}

Keywords: Index model, minimum variance portfolio, maximum sharp portfolio, minimum variance frontiers, minimum return frontiers, efficient frontiers

\section{INTRODUCTION}

According to Hristache [1], An Index Model is a Statistical model of security returns. Bae[2] explained the concept using the effect of three mile island on electric utility stock prices. Elton[3] explained the model in an international aspect. They investigated the international pricing of risk, which is an empirical investigation of the World Capital Market structure. Schneeweis[4] used the Index model in conducting a study of the rise of partial least squares structural equation modeling to explain the application in banking system. Banda, Hall and Pradhan[5], explained the simple criteria for optimal portfolio selection and pointed out the reasons of why portfolio theory has not been implemented.

Francis[6] used the Index Model to estimate the covariance matrix of stock returns by an optimally weighted average of two existing estimators: the sample covariance matrix and single-index covariance matrix. Wolf and Ledoit[7] used Index Model to examine that in the UK, the impact of interest rate changes on the common stock returns of financial institutions' portfolios. Booth[8] reexamined the interest rate sensitivity of common stock returns of financial firms in his article about how interest rate and common stock interact. Solnik[9] used the Index model in his study to investigate the issue of financial intermediaries' common stock returns. Staikouras[10] used Index model in their explanation of industry returns.

For this research project, we focused on using the Index model to measure the Weights, Minimum Variance Portfolio, Maximum Sharpe Portfolio for the ten stocks under different constraints. We will show a complete calculation of maximum Sharpe portfolio and minimum variance portfolio. This allows investors to optimize their portfolios with the given resources. Based on a recent 20-year historical monthly total return data, we will present an investment portfolio analysis by calculating optimization inputs and permissible portfolio regions. Optimization analysis is significant for those who are risk-takers that seek higher return, and for those who likes stable outcomes. Under five constraints, the results we get will be more related to the real finance market.

In this paper, we will first introduce the Index Model itself and how it works, then we will explain how we get the data and interpret the data. After that, we will explain the establishment of portfolios and different 
constraints. Finally, we will analyze the results thoroughly based on the data we got.

\section{INDEX MODEL FOR PORTFOLIO ESTABLISHMENT}

The model we used to measure both the risk and the return of the stocks is the Index Model. This asset pricing model marks the returns on a security to be represented as a linear relationship with any economic variable relevant to the security. And in this case, for stocks, this single factor is the market return. A Single Index Model describes two sources of uncertainty for a security's return. One is systematic uncertainty, which is assumed to be well represented by a single index of stock returns; the other one is Unique uncertainty, which is represented by a security-specific random component.

$$
r(s)-r(f)=\alpha+\beta \times\left(r_{m}-r_{f}\right)+e(1)
$$

According to this formula, alpha represents the abnormal returns for the stock. $\beta(\mathrm{rm}-\mathrm{rf})$ represents the movement of the market modified by the stock's beta. rf represents the risk free rate. $\varepsilon$ represents the unsystematic risk of the security due to firm-specific factors. The situation of the market is reflected in beta, and it affects the asset's returns. Asset returns contain both the firm specific excess returns, which is reflected in alpha and also the firm-specific risk, which is reflected in the residual.

An example of how to use the index Model from Stern, New York University is that when they choose the S\&P500 as the market proxy, they analyse the stock of General Electric (GE), and find that, by using weekly returns, $\alpha \mathrm{j}=-0.07 \%, \beta \mathrm{j}=1.44$. Therefore, when they expect the S\&P 500 to increase by $5 \%$ next week, according to the index model, they would expect the return on GE next week to be $7.13 \%$.

We used the similar way. We counted the Weights, Minimum Variance Portfolio, Maximum Sharpe Portfolio for the ten stocks under different constraints.

When there is no constraint, Minimum Variance Portfolio has a return of $7.152 \%$, a STD of $9.634 \%$, and a Sharpe ratio of 0.88609 .

When it's under Constraint 1 - Regulation $\mathrm{T}$, Minimum Variance Portfolio has a return of $7.152 \%$, a STD of $9.634 \%$, and a Sharpe ratio of 0.74234 .

When it's under Constraint 2 - Arbitrary Box, Minimum Variance Portfolio has a return of $7.152 \%$, a STD of $9.634 \%$, and a Sharpe ratio of 0.74234 .

When it's under Constraint $3-\mathrm{Wi}>0$, Minimum Variance Portfolio has a return of $8.640 \%$, a STD of $10.164 \%$, and a Sharpe ratio of 0.850 .
When it's under Constraint $4-\mathrm{Wi}=0$, Minimum Variance Portfolio has a return of $7.820 \%$, a STD of $9.754 \%$, and a Sharpe ratio of 0.802 .

\section{DATA COLLECTION}

To pursue consistent investment growth in the long term, investment on a set of assets or portfolio could achieve the expectation of return growth. To form a well-diversified portfolio, it could consist of investments in different forms of assets such as stocks, bonds, and securities; and it could be a combination of assets that from completely different sectors. Determining the allocation of assets in the portfolio is the key to investors' returns and profits. For the following analysis, we would use 20 years of historical daily total return data for the S\&P 500 index and for 10 chosen stocks, representing four different sectors according to Yahoo!finance. Table 1 shows the full name of the 10 stocks we would analyse and their belonging sectors. For the following calculations, the data frame for the full available historical data would be started from 5/11/2001 and ended in 5/12/2021.

Table 1.Ticker Symbols, Full Name and Sectors of 10 Chosen Stocks

\begin{tabular}{|c|c|c|}
\hline $\begin{array}{l}\text { Ticker } \\
\text { Symbol }\end{array}$ & Full Name & $\begin{array}{c}\text { Sector } \\
\text { (Yahoo!finance) }\end{array}$ \\
\hline \multirow[t]{2}{*}{$P G$} & The Procter \& & Consumer \\
\hline & Gamble Company & Defensive \\
\hline \multirow[t]{2}{*}{$C L$} & Colgate-Palmolive & Consumer \\
\hline & Company & Defensive \\
\hline \multirow[t]{2}{*}{$A L L$} & The Allstate & Financial Services \\
\hline & Corporation & \\
\hline \multirow[t]{2}{*}{ GS } & The Goldman & Financial Services \\
\hline & Sachs Group, Inc. & \\
\hline \multirow[t]{2}{*}{$T D C N$} & The Toronto- & Financial Services \\
\hline & Dominion Bank & \\
\hline USB & U.S. Bancorp & Financial Services \\
\hline \multirow[t]{2}{*}{ JNJ } & Johnson \& & Healthcare \\
\hline & Johnson & \\
\hline \multirow[t]{2}{*}{$N V D A$} & NVIDIA & Technology \\
\hline & Corporation & \\
\hline $\operatorname{CscO}$ & Cisco Systems, Inc. & Technology \\
\hline INTC & Intel Corporation & Technology \\
\hline
\end{tabular}

Fig.1 depicts the stock prices change of 10 chosen stocks over the 20 years period. Historical stocks prices could be a tool that the investors understand and outlook a stock, where a fundamental analysis on the historical prices could provide some evidence and perceptions on 
company's past performances and its prospect in the future. Fig.2 shows the rate of return, quoted as a percentage, of the chosen 10 stocks over the 20 years period. It represents that, in relation to the initial cost, the return investors could expect in the given time period.

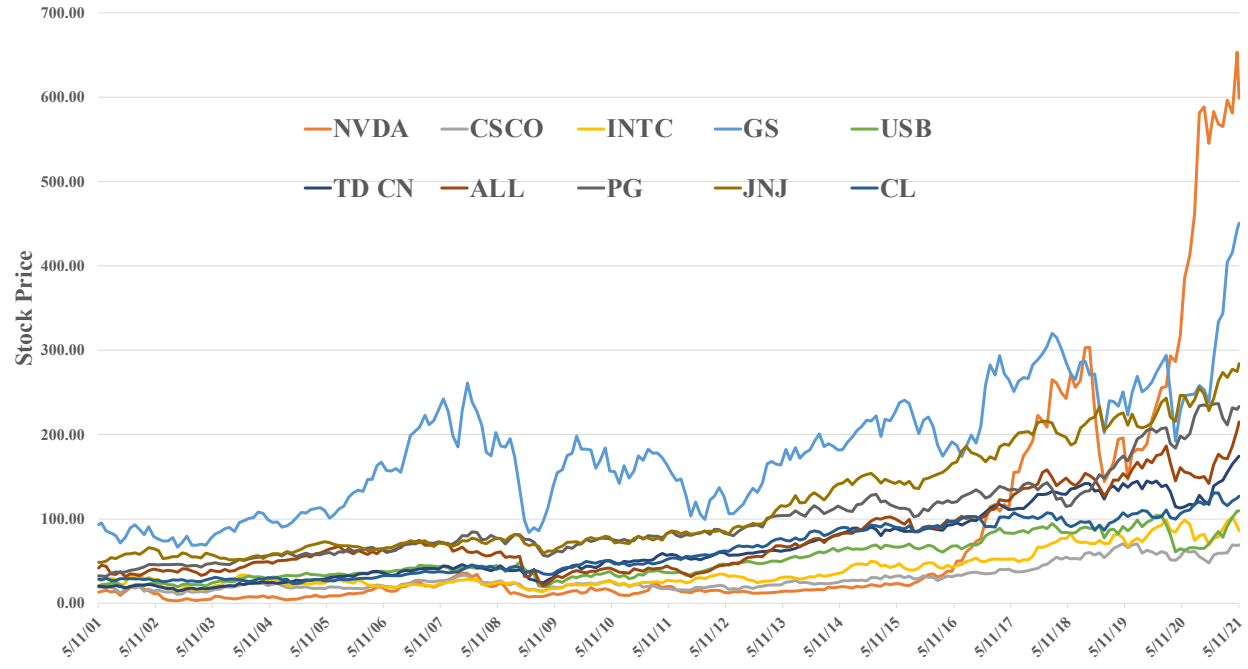

Fig. 1 Stock Prices of 10 Stocks over 20 Years

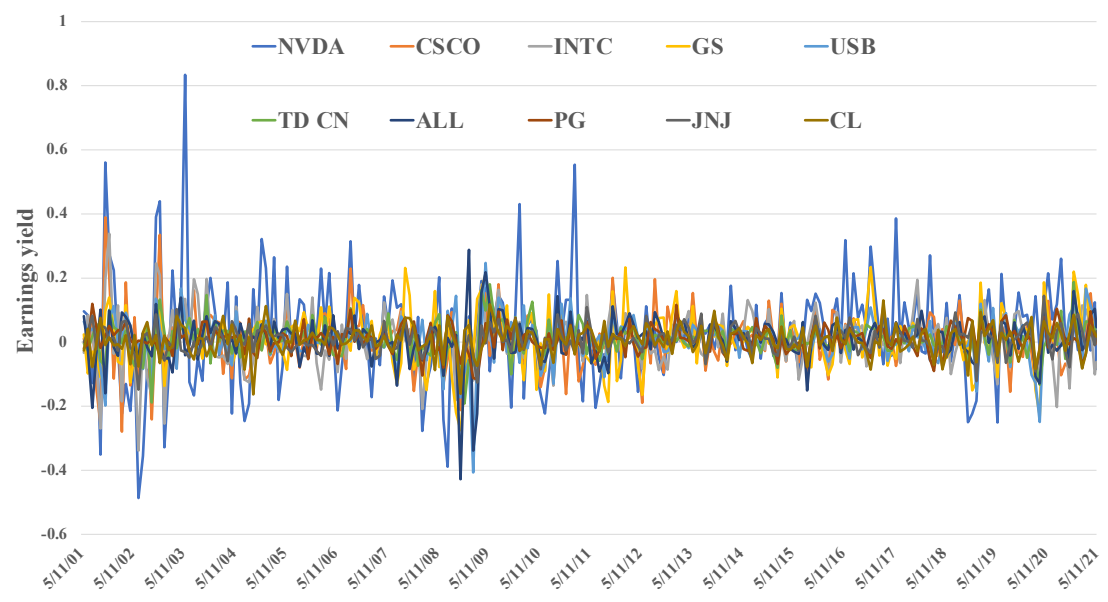

Fig. 2 Rate of Return of 10 Stocks over 20 Years

Moreover, risk-free rate would be represented by the 1-month annual Fed Funds rate, ticker symbol FEDL01. For the sake of eliminating the non-Gaussian effects, we would aggregate the data for 10 stocks from daily total returns to monthly, and then calculate all appropriate optimization inputs for the Index Model, and the permissible portfolios with several specific constraints.

From Table 2, we also conduct a correlation table of monthly returns of the 10 stocks over 20 years and the S\&P 500. Correlation matters significantly when we conduct portfolio investment analysis. A portfolio that highly correlates displays higher risk, meaning that the whole portfolio would possibly fall by the similar amount when one of the stocks falls. Even though risk could not be eliminated, however, possessing a less correlated portfolio means that the overall volatility of the portfolio reduces.

\section{PORTFOLIO ESTABLISHMENT}

In this section, we are going to use index model to generate different optimal portfolio under different constraints. In particular, under each constraint, we will calculate its minimum variance portfolio and maximum Sharpe portfolio, also plotting its minimum variance frontier, minimum return frontier and efficient frontier. In the last part, we will analyse the results of our model, compare the differences between different constraints, and provide investment advice to investors under different constraints. 
Table 2: Correlation of the Monthly Return of 10 Stocks \& SPX

\begin{tabular}{cccccccccccccc}
\hline & SPX & NVDA & CSCO & INTC & GS & USB & TD CN & ALL & PG & JNJ & CL \\
\hline SPX & 1 & 0.527 & 0.637 & 0.578 & 0.708 & 0.609 & 0.645 & 0.630 & 0.412 & 0.542 & 0.440 \\
NVDA & 0.527 & 1 & 0.487 & 0.524 & 0.343 & 0.160 & 0.338 & 0.157 & 0.060 & 0.165 & 0.069 \\
CSCO & 0.637 & 0.487 & 1 & 0.614 & 0.487 & 0.328 & 0.410 & 0.297 & 0.220 & 0.239 & 0.165 \\
INTC & 0.578 & 0.524 & 0.614 & 1 & 0.411 & 0.280 & 0.412 & 0.286 & 0.136 & 0.325 & 0.110 \\
GS & 0.708 & 0.343 & 0.487 & 0.411 & 1 & 0.472 & 0.494 & 0.417 & 0.173 & 0.296 & 0.203 \\
USB & 0.609 & 0.160 & 0.328 & 0.280 & 0.472 & 1 & 0.539 & 0.540 & 0.336 & 0.234 & 0.218 \\
TD CN & 0.645 & 0.338 & 0.410 & 0.412 & 0.494 & 0.539 & 1 & 0.417 & 0.231 & 0.273 & 0.212 \\
ALL & 0.630 & 0.157 & 0.297 & 0.286 & 0.417 & 0.540 & 0.417 & 1 & 0.346 & 0.452 & 0.407 \\
PG & 0.412 & 0.060 & 0.220 & 0.136 & 0.173 & 0.336 & 0.231 & 0.346 & 1 & 0.494 & 0.483 \\
JNJ & 0.542 & 0.165 & 0.239 & 0.325 & 0.296 & 0.234 & 0.273 & 0.452 & 0.494 & 1 & 0.527 \\
\hline
\end{tabular}

\subsection{Constraints translation and portfolio establishment}

\subsubsection{General methods to solve the model}

Different constraints as we have in each model, the general methods to calculate the results are very much the similar.

Firstly, we use the solver in excel to solve for the minimum variance portfolio and maximum Sharpe portfolio. The two problems about solving for two optimal portfolios can be described as programming problems as follow.

$$
r_{p}^{e}=\sum_{i=1}^{11} w_{i} \cdot \beta_{i} \cdot r_{1}^{e}+w_{i} \cdot \alpha_{i}
$$

Where $r_{p}^{e}$ refers to excess return for portfolio, $w_{i}$ represents weight of asset $\mathrm{i}$ in portfolio, $\beta_{\mathrm{i}}$ represents $\beta$ for asset $\mathrm{i}, r_{1}^{e}$ represents excess return for $\mathrm{S} \& \mathrm{P} 500$ index, $\alpha_{i}$ represents $\alpha$ for asset $\mathrm{i}$

$$
\min \sigma_{\mathrm{p}}^{2}=\sum_{\mathrm{i}=1}^{11} \mathrm{w}_{\mathrm{i}}^{2} \cdot \beta_{\mathrm{i}}^{2} \cdot \sigma_{1}^{2}+\mathrm{w}_{\mathrm{i}}^{2} \cdot \varepsilon_{\mathrm{i}}^{2}
$$

Where $\sigma_{p}^{2}$ represents variance for portfolio, $\sigma_{1}^{2}$ represents variance for $\mathrm{S} \& \mathrm{P} 500$ index, $\varepsilon_{i}^{2}$ represents residual variance for asset $\mathrm{i}$.

$$
\max \mathrm{SR}_{\mathrm{p}}=\frac{\mathrm{r}_{\mathrm{p}}^{\mathrm{e}}}{\sigma_{\mathrm{p}}}
$$

Where $S R_{p}$ represents Sharpe ratio for the portfolio, $\sigma_{p}$ represents standard deviation for the portfolio.

Then we will have to implement the solving process in excel. We set the portfolio standard deviation as objective and set the objective to minimum, select the weights of assets in portfolio as changing variable, and then run the solver. We will get minimum variance portfolio in this way. Similarly, we set the portfolio Sharpe Ratio as objective, set objective to maximum, and repeat previous steps, we will have the maximum Sharpe portfolio in this method.

Secondly, we use the solver table to generate the data we used to plot the three frontiers. To run the solver table, we firstly create a dummy variable in excel and let average return of the portfolio equal to it. Then we run the solver table and let it change the dummy variable. By implementing this step, we generate the data used to plot minimum variance frontiers. In the same way, we can let standard deviations equal to dummy variable and use solver table to change the dummy variable, so that we can produce the data used to plot minimum return frontiers and efficient frontiers.

Finally, when we are going to solve the model under a specific restraint, we will have to add restraints to solver, and repeat the previous steps. So, in next context, we will focus on how to translate the real-world regulations into constraints that can be added to models. 


\subsubsection{Regulation $T$}

This additional optimization constraint is designed to simulate the Regulation $\mathrm{T}$ by FINRA, which allows broker-dealers to allow their customers to have positions, $50 \%$ or more of which are funded by the customer's account equity. We translate this regulation into equation:

$$
\text { s.t. } \sum_{i=1}^{11}\left|w_{i}\right| \leq 2
$$

Where $w_{i}$ represents the weights for asset $\mathrm{i}$.

\subsection{3, Arbitrary box}

This additional optimization constraint is designed to simulate some arbitrary "box" constraints on weights, which may be provided by the client:

$$
\text { s.t. }\left|w_{i}\right| \leq 1, \text { for } \forall i
$$

\subsubsection{Free}

The model under no constraints is implemented to how the area of permissible portfolios in general and the efficient frontier in particular look like if you have no constraints.

\subsubsection{No short position}

This additional optimization constraint is designed to simulate the typical limitations existing in the U.S. mutual fund industry: a U.S. open-ended mutual fund is not allowed to have any short positions, which means:

$$
\text { s.t. } w_{i} \geq 0, \text { for } \forall i
$$

\subsubsection{No index}

Lastly, we would like to see if the inclusion of the broad index into our portfolio positive or negative effect has, for that we would like to consider an additional optimization constraint:

$$
\text { s.t. } \mathrm{w}_{1}=0
$$

where $w_{1}$ is the weights for $\mathrm{S} \& \mathrm{P} 500$ index in portfolios

\subsection{Results analysis and comparison}

The results for our models contain two optimal portfolios and three frontiers in one chart for under each constraint.

\subsubsection{Optimal portfolios}

According to previous context, we have five minimum variance portfolio and five maximum Sharpe portfolios in total. After generating the optimal portfolios, we are going to do some analysis about the results in Table 3 and Table 4. First of all, for stocks like CSCO and INTC, no matter under what constraints, they still have negative or 0 weights in both of the portfolios, which indicates that they are not good investments because they are poor in return and huge in risk. In addition, the stock like GS,ALL and USB, although they have positive weights in some portfolio, generally speaking, the absolute value of their weights is so tiny that they are allocated with 0 weights under constraints 4.Therefore, GS,ALL and USB are trivial assets in the portfolio. In contrast $\mathrm{CN}, \mathrm{PG}, \mathrm{JNJ}$ and $\mathrm{Cl}$ are main components of portfolios under every constraint, which means they are good investments with high rate of return and low risk.

Table 3. Weights Under Minimum Variance Portfolio with 5 Constraints

\begin{tabular}{cccccccccccc}
\hline & SPX & NVDA & CSCO & INTC & GS & USB & TDCN & ALL & PG & JNJ & CL \\
\hline Constraint 1 & 0.256 & -0.040 & -0.053 & -0.028 & -0.087 & 0.008 & 0.103 & -0.014 & 0.313 & 0.277 & 0.266 \\
Constraint 2 & 0.256 & -0.040 & -0.053 & -0.028 & -0.087 & 0.008 & 0.103 & -0.014 & 0.313 & 0.277 & 0.266 \\
Constraint 3 & 0.256 & -0.040 & -0.053 & -0.028 & -0.087 & 0.008 & 0.103 & -0.014 & 0.313 & 0.277 & 0.266 \\
Constraint 4 & 0 & 0 & 0 & 0 & 0 & 0 & 0.092 & 0 & 0.336 & 0.289 & 0.283 \\
Constraint 5 & 0 & -0.033 & -0.033 & -0.011 & -0.060 & 0.033 & 0.142 & 0.011 & 0.341 & 0.315 & 0.295 \\
\hline
\end{tabular}


Table 4. Weights Under Maximum Sharpe Portfolio with 5 Constraints

\begin{tabular}{lccccccccccc}
\hline & SPX & NVDA & CSCO & INTC & GS & USB & TD CN & ALL & PG & JNJ & CL \\
\hline Constraint 1 & -0.477 & 0.089 & -0.012 & -0.005 & -0.006 & 0.067 & 0.296 & 0.046 & 0.440 & 0.334 & 0.230 \\
Constraint 2 & -0.702 & 0.103 & -0.006 & -0.001 & 0.006 & 0.094 & 0.343 & 0.074 & 0.469 & 0.369 & 0.251 \\
Constraint 3 & -0.702 & 0.103 & -0.006 & -0.001 & 0.006 & 0.094 & 0.343 & 0.074 & 0.469 & 0.369 & 0.251 \\
Constraint 4 & 0 & 0.067 & 0 & 0 & 0 & 0 & 0.178 & 0 & 0.373 & 0.227 & 0.154 \\
Constraint 5 & 0 & 0.077 & -0.058 & -0.045 & -0.068 & 0.026 & 0.228 & 0.005 & 0.388 & 0.267 & 0.181 \\
\hline
\end{tabular}

Table 5.Sharpe Ratio under Minimum Variance Portfolio \& Maximum Sharpe Portfolio with 5 Constraints

\begin{tabular}{ccccccc}
\hline & \multicolumn{2}{c}{ Rate of Return } & \multicolumn{2}{c}{ Standard Deviation } & \multicolumn{2}{c}{ Sharpe Ratio } \\
\cline { 2 - 6 } & Min.Variance & Max.Sharpe & Min.Variance & Max.Shar & Min.Variar & Max.Shar \\
\hline Constraint 1 & $7.15 \%$ & $12.07 \%$ & $9.63 \%$ & $12.18 \%$ & 0.742 & 0.990 \\
Constraint 2 & $7.15 \%$ & $12.87 \%$ & $9.63 \%$ & $12.92 \%$ & 0.742 & 0.996 \\
Constraint 3 & $7.15 \%$ & $12.87 \%$ & $9.63 \%$ & $12.92 \%$ & 0.742 & 0.996 \\
Constraint 4 & $8.64 \%$ & $10.71 \%$ & $10.16 \%$ & $11.72 \%$ & 0.850 & 0.914 \\
Constraint 5 & $7.82 \%$ & $10.84 \%$ & $9.75 \%$ & $11.48 \%$ & 0.802 & 0.944
\end{tabular}

Different from previous stocks 500 index and NVDA performs differently under different constraints. S\&P 500 index is allocated with positive or 0 weights in minimum variance portfolio and is allocated with negative or 0 weights in maximum Sharpe portfolio. This is because S\&P 500 index is low in risk but also low in rate of return, making it a good investment to minimum the risk(minimum variance portfolio), but a bad investment when considering both rate of return and risk. Different from S\&P 500 index, NVDA stock is high has positive or 0 weights in maximum Sharpe portfolio and negative or 0 weights in minimum variance portfolio. This is because it is high in rate of return but also high in risk. Therefore, it would be a good investment for investors who like taking risks, and a bad investment for a risk averse investor.

No matter what kinds of constraints we add to the model, the optimal portfolios under constraints are always outperformed by those under no constraints. In Table 5, The minimum variance portfolios will have higher variance and maximum Sharpe portfolios will have lower Sharpe ratio under constraints. This phenomenon indicates that regulations aiming at forcing investors to control their risks will eventually have the opposite effects and prevent them from minimizing their risk. This is partly because adding constraints to investors will distort their behaviour and let their decisions become less efficient.

However, Table 5 also indicate that results under constraint 1 and 2 actually are better than those under constraints 3 and 4 ,since they are closer to results with no constraints. This phenomenon indicates that regulations aim at preventing investors making extreme decisions(Constraint 1 and 2) works better than regulations simply ban shorting(regulation 4) and buying index(regulation 5),which, in particular, means minimum variance portfolios under constraints 1 and 2 have smaller variance and maximum Sharpe portfolios have higher Sharpe ratio compared with those under constraints 4 and 5.This can be explain by the fact that optimal portfolios for series of assets will always ask for short positions in some assets and preventing investors from having short positions will ruin their optimal decisions. 


\subsubsection{Frontiers and charts}

To make the difference between models under different constrains more specific and straightforward, we generate a figure containing minimum variance frontier(MVF), minimum return frontier(MRF), and efficient frontier(EF) under each constrains.

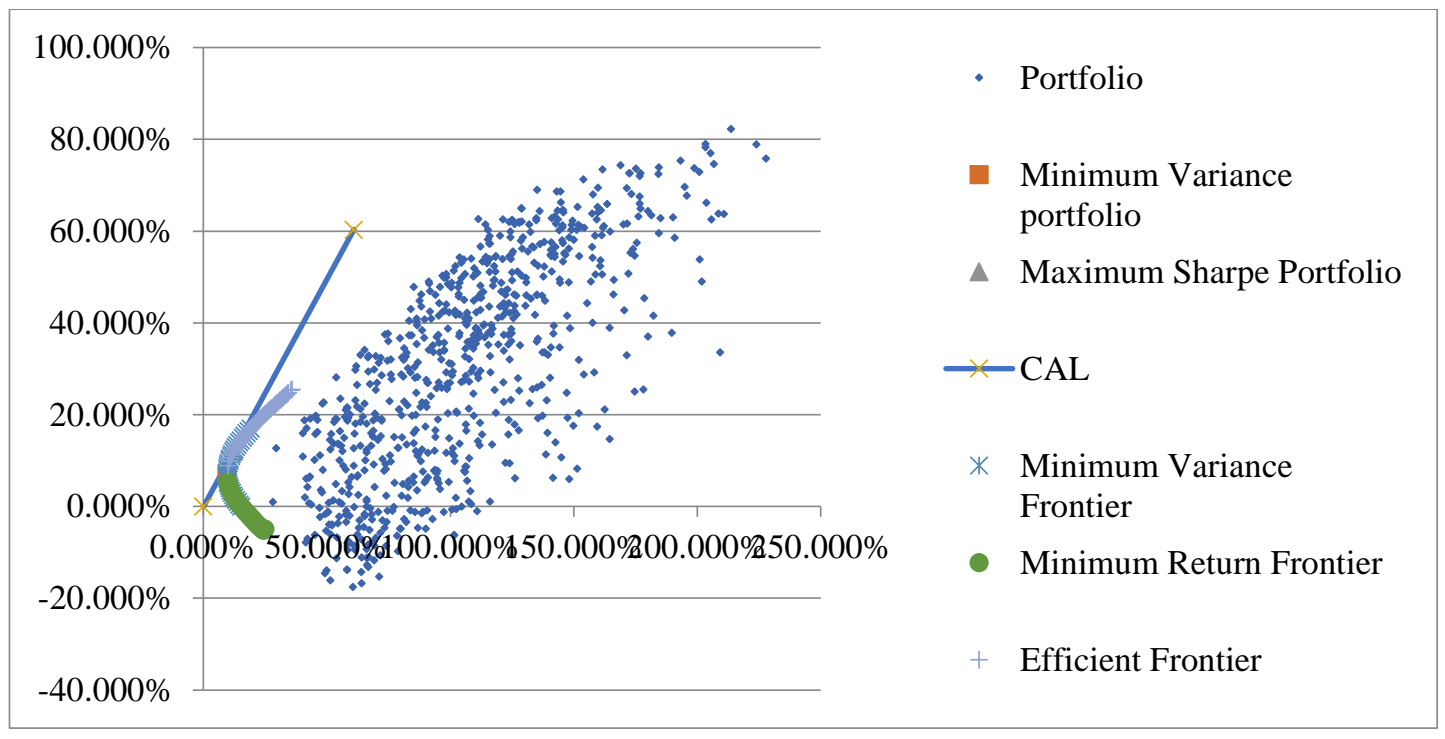

Fig. 3 Index Model with Constraint 1 - Regulation T



Fig. 4 Index Model with Constraint 2 - Arbitrary "Box"

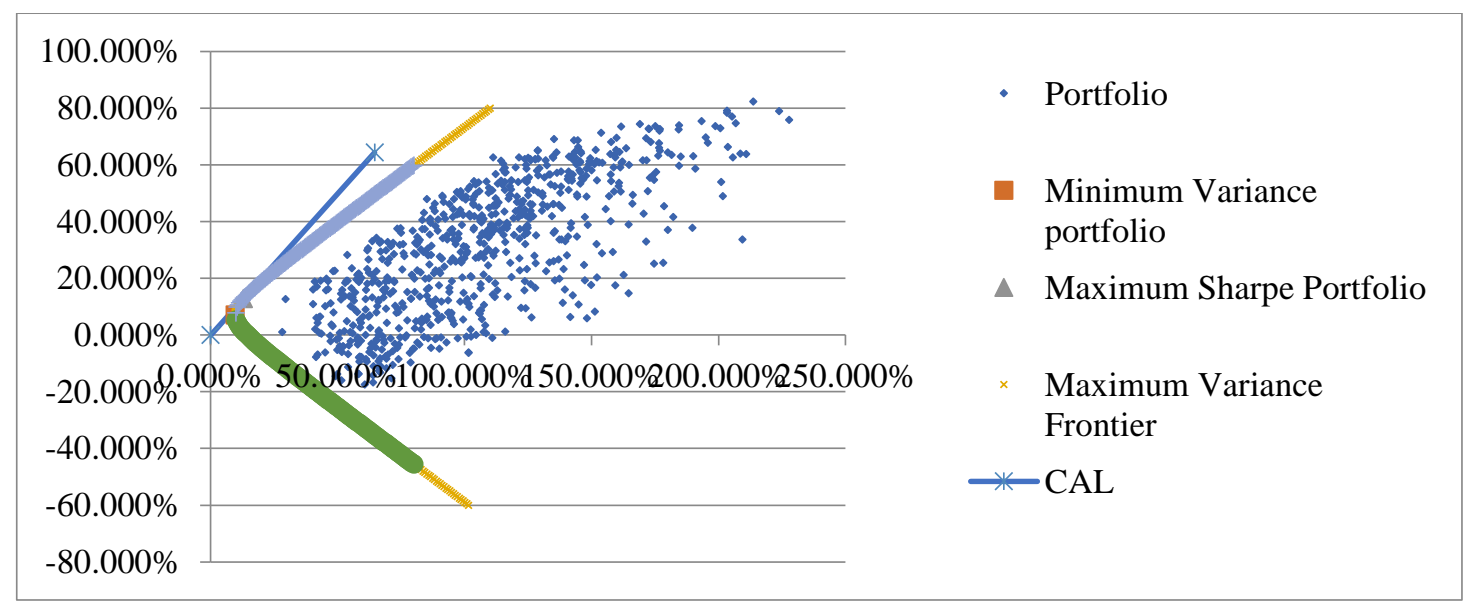

Fig. 5 Index Model with Constraint 3 - "Free" 




Fig. 6 Index Model with Constraint 4 - No Short Positions



Fig.7 Index Model with Constraint 5 - Inclusion of Broad Index

As it is showed in the charts above, the consistence of 5 figures is similar. The MVF is the main curve of the figure. The upper side of MVF is EF and the other side is MRF.EF and capital allocation line(CAL) are tangent, and the tangency point is MS. The peak for MVF is MVP. Although the figures under different constraints have many differences, but they all consist of these basic parts.

The main difference between charts is the shape of curves. In Fig.5 and Fig.7, 3 frontiers under constraint 5 are very much the similar with those under constraint 3 compare with others. This is because constraint 5 are just simply removing S\&P 500 index from portfolios and remaining 10 stocks still can form a normal chart. Charts under constraint 4 is the most different one from the that without constraints.

As it is showed in the Fig.6, The MVF,MRF and EF are becoming almost the same curve and the lower side of MVF disappears. This is mainly because constraint 4 are too strict that there is only one solution under such constraints. Therefore no matter what objective we select and whether we set objective to minimum or maximum, we will get the same solution.

In Fig.3 and Fig.4,the other two charts are slightly different from the chart under no constraints. This is because constraint 1 and 2 do not eliminate all possible short position, they just simply restrict investors to have extreme weights on some assets. In this way, they distort investor behaviour less than constraint 4 .

\section{CONCLUSION}

We have presented an investment portfolio analysis based on a recent 20 years of historical monthly total return data for 10 chosen stocks by calculating optimization inputs and permissible portfolios regions using Index Model. We also presented a full calculation of minimum variance portfolio and maximum Sharpe portfolio, which could provide sources of evidences to investors when optimizing their portfolios. For both investors who are risk-takers seeking higher return, and who expects stable outcomes with low uncertainty, optimization analysis is necessary. Performed under 5 constraints, we intended to make our analysis and conclusion more related to the real complicated finance market, rather than theoretically. We identified the significance and correlation of optimization under each condition. For this analysis, we are focusing on performing Index Model to make an investigation on the investment portfolio with 5 different constraints. In the future, we could further investigate another model, Markowitz Model, to find the correlation and difference 
between the two to get a more comprehensive understanding on investment portfolio.

\section{REFERENCES}

[1] [1] Hristache, M., Juditsky, A., \& Spokoiny, V. (2001). Direct Estimation of the Index Coefficient in a Single-Index Model. The Annals of Statistics, 29(3), 595-623. Retrieved August 30, 2021, from http://www.jstor.org/stable/2673964.

[2] S. Bae, "Interest Rate Changes and Common Stock Returns of Financial Institutions: Revisited.” Wiley Online Library, John Wiley \&amp; Sons, Ltd, 27 Aug. 2014 , onlinelibrary.wiley.com/doi/abs/10.1111/j.14756803.1990.tb00537.x.

[3] E.J. Elton, et al. "Simple Criteria for Optimal Portfolio Selection." The Journal of Finance, vol. 31, no. 5, 1976, pp. 1341-1357. JSTOR, www.jstor.org/stable/2326684. Accessed 26 Aug. 2021.

[4] J. Hill, T.Schneeweis, "The Effect of Three Mile Island on Electric Utility Stock Prices: A Note." "The Journal of Finance", vol. 38, no. 4, 1983, pp. 1285-1292.

JSTOR, www.jstor.org/stable/2328026. Accessed 26 Aug. 2021.

[5] Kamoto Banda, John Henry Hall, Rudra P. Pradhan. (2019) The impact of macroeconomic variables on industrial shares listed on the Johannesburg Stock Exchange. Macroeconomics and Finance in Emerging Market Economies 12:3, pages 270-292.

[6] "Interest Rate Changes and Common Stock Returns of Financial Institutions: Evidence from the UK." T. Francis, www.tandfonline.com/doi/abs/10.1080/135184798 337344 .

[7] O. Ledoit, M. Wolf, "Improved Estimation of the Covariance Matrix of Stock Returns with an Application to Portfolio Selection." Journal of Empirical Finance, North-Holland, 17 Apr. 2003, www.sciencedirect.com/science/article/abs/pii/S09 27539803000070 .

[8] Booth, J. R. and Officer, D. T. 1985. Expectations interest rates and commercial bank stocks. The Journal of Financial Research, 8: 51-58.

[9] B.H. Solnik, "The International Pricing of Risk: An Empirical Investigation of the World Capital Market Structure." vol. 29, no. 2, 1974, pp. 365378. www.jstor.org/stable/2978806. Accessed 26 Aug. 2021.
[10] S. Staikouras, "Equity Returns of Financial Institutions and the Pricing of Interest Rate Risk." T.

Francis, www.tandfonline.com/doi/full/10.1080/096031005 00039557. 